\title{
The Prediction of Maternal Serum Alpha Fetoprotein Level and Fetal Nuchal Translucency, Forcases Involving Preterm Prelabor Rupture of Membranes
}

\section{Yiming Chen ( $\nabla$ cxy40344@163.com )}

Hangzhou Women's Hospital https://orcid.org/0000-0003-1532-6049

\section{Yijie Chen}

Zhejiang Chinese Medical University

\section{Lei Chen}

Hangzhou Children's Hospital

\section{Wenwen Ning}

Zhejiang chinese medical university

\section{Xiaoying Wang}

Hang women's Hospital

\section{Zhifen Zhang}

Hangzhou women's hospital

\section{Research article}

Keywords: pregnancy associated plasma protein A, alpha-fetoprotein, free $\beta$ subunit of human chorionic gonadotropin, nuchal translucency, preterm prelabor rupture of membranes, adverse pregnancy outcomes

Posted Date: January 18th, 2021

DOl: https://doi.org/10.21203/rs.3.rs-146931/v1

License: (c) (i) This work is licensed under a Creative Commons Attribution 4.0 International License. Read Full License 


\section{Abstract}

Objective: To investigate whether the values of maternal serum alpha-fetoprotein (AFP) and fetal nuchal translucency (NT) during the first and second trimesters can predict preterm prelabor rupture of membranes (PPROM).

Methods: This retrospective case-control study analyzed the first and second trimester screening indicators and maternal outcomes of gravidas who were divided into the Non-PPROM group (594) and the PPROM group (591). Binary logistic regression analysis was used to calculate the OR and $95 \% \mathrm{Cl}$. ROC and AUC were used for screening performance of AFP and NT.

Results: The values of NT and AFP in the PPROM group were higher than the control group and the difference was statistically significant (all $P<0.05$ ). There was no significant difference with respect to PAPP-A, free $\beta$ hCG, and the calculated risk of trisomy 18 (all $P>0.05$ ). Binary logistic regression showed that NT and AFP MoM were risk factors for PPROM, with ORs of 1.719 and 3.549, respectively. The AUC according to the ROC for NT, AFP MoM and maternal weight in the first trimester +NT+AFP were 0.552 , 0.618 and 0.630 .

Conclusions: NT and AFP during the first and second trimesters were identified as risk factors for PPROM and valuable markers to predict PPROM in late pregnancy. Multi-index joint prediction was more effective.

\section{Introduction}

The definition of prelabor rupture of membranes(PROM)is rupture of membranes before the onset of labor [1]. Membrane rupture before 37 weeks of gestation is considered as preterm prelabor rupture of

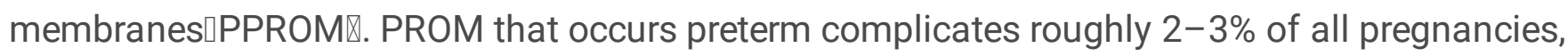
representing a percentage of preterm births, whereas term PROM occurs in approximately $8 \%$ of pregnancies in the United States [2]. Although PROM can result from a normal physiologic weakening of the membranes associated with shearing forces created by uterine contractions, PPROM can result from a wide array of pathologic mechanisms that act individually or in concert [3]. Sepsis, necrotizing enterocolitis and intraventricular hemorrhage also are associated with prematurity. PPROM has been associated with an increased risk of neurodevelopmental impairment [4-5].

AFP is the dominant serum protein early in fetal life, which is synthesized in the fetal liver and yolk sac, reaching a maximum concentration in the in first and second trimester, thereafter, its concentration drops steadily [6]. The combined examination of AFP, PAPP-A, free $\beta-h C G$ and fetal NT has been extensively used to screen fetal Down syndrome (DS), open neural tube defects (ONTD), Edwards syndrome (ES) and other diseases [7-8]. However, there are few researches on the prediction of PPROM for AFP, free $\beta-h C G$, PAPP-A levels, and fetal NT values. In the retrospective studies conducted by Öztürk $\mathrm{H}$ et al., a significant relationship was found between abnormal elevation of hCG and AFP levels and adverse pregnancy outcomes (APO), such as pre-eclampsia, intrauterine growth retardation (IUGR), placenta accreta, PPROM [9]. 
An accurate assessment of gestational age and knowledge of the maternal, fetal risks are important to appropriate evaluation, counseling, and care of patients with PROM. Expectant management in pregnancies complicated by PPROM is related with an overall neonatal survival rate of $49.0 \%$, high risk of considerable maternal risks [10]. The aim of this study was to assess whether AFP and fetal NT are associated with PPROM and provide evidence for predicting PPROM in pregnancy women.

\section{Methods}

\section{Study population}

This retrospective case-control study was conducted in Hangzhou Women's Hospital between January 2015 and April 2020. We analyzed the first and second trimester screening indicators and maternal outcomes of gravidas who were divided into the Non-PPROM group (594) and the PPROM group (591). PPROM was diagnosed according to the elevated vaginal fluid, vaginal power of hydrogen measurement, ultrasound examination et al $[1,11]$. The exclusion criteria were as follows: (1) alcohol abuse and cigarette smoking; (2) special history of pregnancy, such as infants conceived in vitro, plural gestations; (3) co-existing medical conditions, such as chronic hypertension, diabetes mellitus, hyperthyroidism, heart disease, kidney disease, autoimmune disease, blood diseases and a history of immunotherapy or blood transfusion or special medications during pregnancy; (4) DS, ES, ONTD and other congenital birth defects; (5) missing more than 3 incomplete information; After careful screening, The Non-PPROM group were randomly selected according to the number of the PPROM group (1:1). The study group included 591 with PPROM and the control group included 594 pregnant women of normal labor. All participants provided written informed consent before participating in the study. The study was approved by the local Ethics Committee (Reference number: 2018-004-01).

\section{Blood Samples And Screening Indicators}

Take fasting venous blood from pregnant women at 11 weeks to 13 weeks and 6 days of gestation and 15 weeks to 20 weeks and 6 days of gestation. After 30 minutes separation, the serum samples were stored in a refrigerator at $2-8{ }^{\circ} \mathrm{C}$ and were sent for examination within 1 week. Screening indicators and programs: (1) Free $\beta$-hCG, PAPP-A levels and fetal NT thickness by ultrasound in first trimester; (2) Free $\beta$ hCG levels and AFP in second trimester; (3) Joint Screening Program: The results of AFP and free $\beta$-hCG in second trimester were matched with those of PAPP-A and NT in first trimester; and the risk rates of T21 and T18 were calculated.

\section{Reagents And Apparatuses}

The apparatuses are applied in the study included 1235 Automatic Immunoassay System (DELFIA) (PerkinElmer, Shelton, USA) for detection with a single labeling kit (PAPP-A, free $\beta$-hCG) in first trimester and a double labeling kit (AFP/free $\beta-h C G)$ in second trimester, enhancement solution and washing liquor 
as well as the quality control sample and VolusonE8 ultrasonic apparatus (GE, Boston, USA) and the standard (WallacOy, Turku, Finland).

\section{Nt Thickness Measurement}

Fetal NT thickness was screened according to the standards issued by Fetal Medicine Foundation (https://fetalmedicine.org/education/the-11-13-weeks-scan). The fetus was normal in case of NT thickness $<2.5 \mathrm{~mm}$, abnormal in case of NT thickness $\geq 2.5 \mathrm{~mm}$. A midsagittal view of the fetus was taken and in a natural posture, the soft tissues overlying the cervical spine was measured by magnifying the image to display the head and upper chest of the fetus only and the maximum thickness of the translucent space between the skin [12]. NT was measured by ultrasound (VolusonE8, GE) at 11 weeks to 13 weeks and 6 days of gestation.

\section{Definition And Calculation Of The Multiple Of Median (mom)}

The original concentration levels of PAPP-A AFP, free $\beta$-hCG and the values of NT were replaced by MoM value using model analysis and calibrate the MoM values with maternal weight and gestational age [13].

\section{Statistical analysis}

All statistical analyses were performed using IBM SPSS Statistics (version 24.0; Chicago, USA). We used a one-sample Kolmogorov-Smirnov test to test data for normality. Skewed data are expressed as medians and percentiles $\mathrm{M}(\mathrm{P} 2.5-\mathrm{P} 97.5)$. The maternal age and NT values were normally distributed and expressed as mean \pm standard deviation $(x \pm s)$. Independent $t$-test was employed between or among groups. The Mann-Whitney $\mathrm{U}$ test were used to compare two groups. Binary logistic regression analysis was used to calculate the odds ratio $(\mathrm{OR})$ and $95 \%$ confidence interval $(\mathrm{Cl})$ for each variable. The best cut off value and AUC were determined by ROC. Besides, the youden index was calculated. $P<0.05$ was considered to be statistically significant.

\section{Results}

\section{Comparison of demographic data between the Non-PPROM group and the PPROM group}

Maternal weight in the first and second trimester and gestational age at testing in the second trimester were all significantly higher in the PPROM group than the control group $(Z=3.584, P<0.001 ; Z=3.491, P$ $<0.001 ; Z=2.007, P=0.045$; respectively; Table 1). There was no significant difference with respect to maternal age, gestational age at testing in the first trimester between the two groups $(P>0.05)$ 
Table 1

Comparison of demographic data between the non-PPROM group and the PPROM group.

\begin{tabular}{|c|c|c|c|c|}
\hline Groups & $\begin{array}{l}\text { Non-PPROM }(n= \\
594)\end{array}$ & PPROM (n= 591) & tor $Z$ & $P$ \\
\hline Maternal age (years) & $\begin{array}{l}28.40 \pm \\
2.85(28.17- \\
28.63)\end{array}$ & $\begin{array}{l}28.64 \pm \\
2.85(28.40-28.66)\end{array}$ & 1.399 & 0.162 \\
\hline $\begin{array}{l}\text { Maternal weight in the first trimester } \\
(\mathrm{kg})\end{array}$ & $\begin{array}{l}52.00(42.40- \\
68.56)\end{array}$ & $\begin{array}{l}53.50(41.50- \\
70.24)\end{array}$ & 3.584 & $\begin{array}{l}< \\
0.001 * \star\end{array}$ \\
\hline $\begin{array}{l}\text { Maternal weight in the second } \\
\text { trimester }(\mathrm{kg})\end{array}$ & $\begin{array}{l}53.50(43.50- \\
69.41)\end{array}$ & $\begin{array}{l}55.00(43.00- \\
71.30)\end{array}$ & 3.491 & $<.001 * *$ \\
\hline $\begin{array}{l}\text { Gestational age at testing in the first } \\
\text { trimester(days) }\end{array}$ & $\begin{array}{l}90.00(80.00- \\
97.00)\end{array}$ & $\begin{array}{l}90.00(79.00- \\
97.00)\end{array}$ & 0.397 & 0.691 \\
\hline $\begin{array}{l}\text { Gestational age at testing in the } \\
\text { second trimester(days) }\end{array}$ & $\begin{array}{l}117.00(108.00- \\
128.00)\end{array}$ & $\begin{array}{l}118.00(108.00- \\
128.00)\end{array}$ & 2.007 & $0.045^{\star}$ \\
\hline
\end{tabular}

\section{Comparison of screening indicators (MOM) and the calculated risk of T21,T18}

AFP and NT in the PPROM group were $1.03 \mathrm{MoM}$ and $0.96 \mathrm{MoM}$ in Table 2 and these were significantly higher than the Non-PPROM group (0.93MoM, $Z=5.995, P<0.001 ; 0.93 \mathrm{MoM}, t=2.352, P=0.019$, respectively). In addition, the calculated risk of T21 in the PPROM group was 1/15393 and this was higher than the Non-PPROM group $(1 / 13714 ; Z=1.438, P=0.015)$. Conversely, the MoM for maternal PAPP-A, free $\beta-h C G$ and the calculated risk of T18 in the PPROM group did not show any significant differences when compared with the Non-PPROM group. 
Table 2

Comparison of screening indicators (MOM) and the calculated risk of T21,T18

\begin{tabular}{|c|c|c|c|c|}
\hline Groups & Non-PPROM $(n=594)$ & PPROM (n = 591) & $\operatorname{tor} Z$ & $P$ \\
\hline PAPP-A MoM & $0.95(0.33-2.41)$ & $1.00(0.28-2.35)$ & 0.707 & 0.480 \\
\hline $\begin{array}{l}\text { Free } \beta \text {-hCG MoM in the first } \\
\text { trimester }\end{array}$ & $1.07(0.34-3.48)$ & $1.02(0.39-3.44)$ & 0.381 & 0.703 \\
\hline NT MoM & $0.93 \pm 0.25(0.90-0.95)$ & $0.96 \pm 0.24(0.94-0.98)$ & 2.352 & $0.019 *$ \\
\hline AFP MoM & $0.93(0.52-1.72)$ & $1.03(0.55-1.87)$ & 5.995 & $\begin{array}{l}<.001 * * \\
0.00\end{array}$ \\
\hline $\begin{array}{l}\text { Free } \beta \text {-hCG MoM in the } \\
\text { second trimester }\end{array}$ & $1.03(0.34-3.49)$ & $1.02(0.36-3.77)$ & 0.741 & 0.459 \\
\hline Calculated risk of T21 & $\begin{array}{l}1 / 13714(1 / 339- \\
1 / 100000)\end{array}$ & $\begin{array}{l}1 / 15393(1 / 493-1 / 100 \\
000)\end{array}$ & 1.438 & $0.015^{\star}$ \\
\hline Calculated risk of T18 & $\begin{array}{l}1 / 100000(1 / 22092- \\
1 / 100000)\end{array}$ & $\begin{array}{l}1 / 100000(1 / 13914- \\
1 / 100000)\end{array}$ & 0.507 & 0.612 \\
\hline \multicolumn{5}{|c|}{$\begin{array}{l}\text { Data are presented as median (P2.5-P97.5). AFP, a-fetoprotein; hCG, human chorionic gonadotropin; } \\
\text { PAPP-A, pregnancy-associated plasma protein } A ; N T \text {, nuchal translucency; } T 21 \text {, trisomy } 21 ; \mathrm{T} 18, \\
\text { trisomy } 18 ; \text { MoM, multiple of median. }{ }^{\star} P<0.05 ; \star \star P<0.001 \text {. }\end{array}$} \\
\hline
\end{tabular}

\section{Results Of Binary Logistic Regression Analysis}

Binary logistic regression analysis showed that the ORs and 95\% Cls for maternal weight, AFP and NT were 1.030 (95\% Cl:1.011-1.049), 3.549 (95\% Cl:2.323-5.421) and 1.719 (95\% Cl:1.011-2.923) in Table 3, respectively, which were the risk factors of PPROM.

Table 3

Binary logistic analysis of maternal weight in the first trimester, AFP, NT and PPROM

\begin{tabular}{|c|c|c|c|c|c|c|c|c|}
\hline \multirow[t]{2}{*}{ Indicators } & \multirow[t]{2}{*}{$\beta$} & \multirow[t]{2}{*}{ SE } & \multirow[t]{2}{*}{ Wald } & \multirow[t]{2}{*}{ df } & \multirow[t]{2}{*}{$P$} & \multirow[t]{2}{*}{ OR } & \multicolumn{2}{|c|}{$95 \% \mathrm{Cl}$ for OR } \\
\hline & & & & & & & Lower & Upper \\
\hline Weight (kg) & 0.029 & 0.009 & 9.580 & 1 & $0.002^{\star}$ & 1.030 & 1.011 & 1.049 \\
\hline NT(MoM) & 0.542 & 0.271 & 4.002 & 1 & $0.045^{\star}$ & 1.719 & 1.011 & 2.923 \\
\hline AFP(MoM) & 1.267 & 0.216 & 34.336 & 1 & $<0.001^{\star *}$ & 3.549 & 2.323 & 5.421 \\
\hline
\end{tabular}

Predictive value of AFP, NT and maternal age separate screening and joint screening for PPROM 
ROC analysis was performed to determine the predictive diagnosis value of maternal age, NT and AFP for PPROM (see Table 4 and Fig. 1). At the best cutoff level of $0.98 \mathrm{MoM}$, AFP predicted PPROM AUC = $0.618(95 \% \mathrm{Cl}: 0.583-0.653, P<0.001)$, with a sensitivity, specificity and youden index of $0.583,0.603$, and 0.186 , respectively. Besides, at the best cutoff level of $0.91 \mathrm{MoM}, \mathrm{NT}$ accurately predicted PPROM AUC $=$ 0.552 (95\% Cl: $0.516-0.588, P=0.005)$, with a sensitivity, specificity and youden index of $0.579,0.531$, and 0.111 , respectively. After combining AFP, NT and maternal weight, the AUC increased to 0.630 ( $95 \% \mathrm{Cl}$ : $0.595-0.664, P<0.001)$.

Table 4

The value of AFP, NT and maternal age separate screening and joint screening for PPROM.

\begin{tabular}{|c|c|c|c|c|c|c|c|}
\hline Indicators & AUC & $95 \% \mathrm{Cl}$ & $P$ & $\begin{array}{l}\text { cut- } \\
\text { off }\end{array}$ & Sensitivity & Specificity & $\begin{array}{l}\text { Jordan } \\
\text { index }\end{array}$ \\
\hline Weight (kg) & 0.556 & $\begin{array}{l}0.520- \\
0.592\end{array}$ & $0.002^{\star}$ & 52.45 & 0.591 & 0.517 & 0.108 \\
\hline NT(MoM) & 0.552 & $\begin{array}{l}0.516- \\
0.588\end{array}$ & $0.005^{\star}$ & 0.91 & 0.579 & 0.531 & 0.111 \\
\hline AFP(MoM) & 0.618 & $\begin{array}{l}0.583- \\
0.653\end{array}$ & $<.001^{\star *}$ & 0.98 & 0.583 & 0.603 & 0.186 \\
\hline $\begin{array}{l}\text { Weight + NT + } \\
\text { AFP }\end{array}$ & 0.630 & $\begin{array}{l}0.595- \\
0.664\end{array}$ & $<.001^{\star \star}$ & 0.47 & 0.685 & 0.508 & 0.194 \\
\hline
\end{tabular}

\section{Discussion}

There are few researches about fetal NT values, maternal serum screening indicators and PPROM. Therefore, we used a retrospective case-control study method to explore the correlation and predictive value of AFP and NT with PPROM. The result of the study showed that the level of NT, AFP and the calculated risk of T21 in the PPROM group were higher than the Non-PPROM group and the difference was statistically significant $(P<0.05)$ in Table 2 , which is consistent with the report by Başbuğ $D$ et al. Adverse pregnancy outcomes, such as preterm delivery, preterm premature rupture of membranes (PPROM), oligohydramnios and intrauterine growth restriction (IUGR) rates were increased in the elevated AFP group [14]. Conversely, the MoM for maternal PAPP-A and free $\beta$-hCG in the PPROM group did not show any significant differences when compared with the control group $(P>0.05)$. Besides, the results of other researches are similar with our study. There were no differences in any major APO between pregnant women with low free $\beta$-hCG levels and control subjects, including preterm birth, low birth weight at term, intrauterine fetal death, abruptio placentae, preeclampsia, or PPROM [15-16]. Contrary to our study, low maternal serum PAPP-A levels during the first trimester may reflect a trophoblast invasion defect in the maternal-fetal interface, resulting in subsequent preterm delivery in a retrospective study, particularly PPROM [17]. 
Binary logistic regression analysis showed that the ORs and AUC of AFP and NT were 3.549, 0.618 and $1.719,0.552$. However, the AUC of Weight + AFP + NT was 0.630 in Table 3 and Table 4, which presented that multi-index prediction combining AFP, NT and maternal age is more effective. The previous researches just mention PPROM as one outcome of adverse pregnancy. There was an established association between an inflammatory intrauterine environment and APO which is independent of gestational age and mediated by the fetal inflammatory response [18]. A recent morphological study showed that inflammation/infection and malperfusion were the two most common pathological changes in the placentas of preterm birth [19]. Placental inflammation did not increase the expressions of AFP in maternal liver, fetal liver and placenta, but reduced the fetal serum AFP levels in a rat model. Placental inflammation can be the potential cause linking APO and AFP [20]. However, there are limitations to this study. Most studies of second-trimester and PPROM are retrospective and include only expectantly managed cases because of selection bias. The basic mechanisms are unclear at present. Therefore, further studies are required to validate and confirm the findings of this study.

The pregnant women in the PPROM group have higher levels of maternal weight in the first and second trimester and the gestational age at testing in the second trimester than women in the Non-PPROM group $(P<0.05$, Table 1$)$. Maternal obesity is associated with increased systemic inflammation and an increased risk of preterm premature rupture of membranes [18]. A retrospective cohort study presented that a higher risk for preterm premature rupture of the membranes at the upper extremes of BMI (OR 1.6; $95 \% \mathrm{Cl}=1.1-2.3 ; P=0.02$ ) [21]. In a population-based retrospective cohort study, the incidence rate of PROM was correlated inversely with body mass index (BMI) and waist circumference. We considered that their low BMI $(<17.5$ and $17.5-19.9 \mathrm{~kg} / \mathrm{m})$ and low WC $(<60$ and $60.0-64.0 \mathrm{~cm})$ groups were different from the present study [22].

\section{Conclusion}

In conclusion, the increase of AFP level, NT and maternal weight are risk factors of PPROM, which may be helpful for the prediction of PPROM in the pregnant women. However, PAPP-A and free $\beta$-hCG in first and second trimester had no association with PPROM.

\section{Abbreviations}

PAPP-A: pregnancy-associated plasma protein A; free- $\beta$ hCG: free beta human chorionic gonadotropin; NT: nuchal translucency; PPROM: predict preterm prelabor rupture of membranes; MoM: multiple of the median; ROC: receiver operating characteristic; AUC: area under curve; OR: Odds ratio.

\section{Declarations}

\section{Conflict Of Interest}

The authors have no conflicts of interest to declare. 


\section{Statement Of Ethics}

The study was approved by Hangzhou Women's Hospital (Hangzhou Maternity and Child Health Care Hospital) ethics committee, in accordance with the ethical standards as laid down in the 1964 Declaration of Helsinki and its later amendments or comparable ethical standards. And the approval number was [2018] medical ethics (004) No.01. Consent you obtained from study participants was written.

\section{Data Availability Statement}

Data sharing is not applicable to this article as no new data were created or analyzed in this study.

\section{Funding}

This study was supported by the Zhejiang Public Welfare Technology Research Program/Social Development (Grant number: LGF19H040006); Medical and Health Research Project of Zhejiang province (grant number 2021KY258); Hangzhou Science and Technology Program Guide Project/Agriculture and Social Development (grant number 20181228Y13).

\section{Acknowledgments}

The authors wish to acknowledge the assistance of Songhe Chen from the medical records room of Hangzhou Women's Hospital in case collection and data matching. Thank to Xiao Lu of the Data Analysis Department of Zhejiang Biosan Biochemical Technologies Co., Ltd.

\section{Authors' Contributions}

Y. M. Chen, Y. J. Chen, and Z. F. Zhang conceptualized and refined the study design and methodology. W. W. Ning conducted data collection and tested blood samples and screening indicators. Y. M. Chen and X.Y. Wang conducted all analyses and prepared tables and figures with assistance from Data Analysis Department. L. Chen assessed the ultrasound of fetal nuchal translucency. Y. J. Chen wrote the entire manuscript and performed literature review. All authors read and approved the final manuscript.

\section{References}

1. Prelabor Rupture of Membranes. ACOG Practice Bulletin, Number 217. Obstet Gynecol. 2020;135(3):e80-97.

2. Bond DM, Middleton P, Levett KM, et al. Planned early birth versus expectant management for women with preterm prelabourrupture of membranes prior to 37 weeks' gestation for improving pregnancy outcome. Cochrane Database Syst Rev.2017;3CD4735.

3. Moore RM, Mansour JM, Redline RW, et al. The physiology of fetal membrane rupture: insight gained from the determination of physical properties. Placenta. 2006;27(11-12):1037-51. 
4. Drassinower D, Friedman AM, Obican SG, et al. Prolonged latency of preterm prelabour rupture of membranes and neurodevelopmental outcomes: a secondary analysis. BJOG. 2016;123(10):162935 .

5. Quist-Nelson J, de Ruigh AA, Seidler AL, et al. Immediate Delivery Compared With Expectant Management in Late Preterm Prelabor Rupture of Membranes: An Individual Participant Data Metaanalysis. Obstet Gynecol. 2018;131(2):269-79.

6. Palomaki GE, Bupp C, Gregg AR, et al. Laboratory screening and diagnosis of open neural tube defects, 2019 revision: a technical standard of the American College of Medical Genetics and Genomics (ACMG). Genet Med. 2020;22(3):462-74.

7. Alldred SK, Takwoingi Y, Guo B, et al. First trimester ultrasound tests alone or in combination with first trimester serum tests for Down's syndrome screening. Cochrane Database Syst Rev.2017;3(D12600.

8. Wald NJ, Huttly WJ, Hackshaw AK. Antenatal screening for Down's syndrome with the quadruple test. LANCET. 2003;361(9360):835-6.

9. Ozturk H, Erkaya S, Altinbas $S$, et al. The role of unexplained high serum alpha-fetoprotein (AFP) and human chorionic gonadotropin ( $\mathrm{hCG}$ ) levels in the second trimester to determine poor obstetric outcomes. Turk J Obstet Gynecol. 2014;11(3):142-7.

10. Kibel M, Asztalos E, Barrett J, et al. Outcomes of Pregnancies Complicated by Preterm Premature Rupture of Membranes Between 20 and 24 Weeks of Gestation. Obstet Gynecol. 2016;128(2):31320.

11. Robert Ehsanipoor. Premature Rupture of Membranes: ACOG Practice Bulletin, Number 139. Obstet Gynecol. 2013;122(4):918-30.

12. Snijders RJ, Noble P, Sebire N, Souka A, Nicolaides KH. UK multicentre project on assessment of risk of trisomy 21 by maternal age and fetal nuchal-translucency thickness at 10-14 weeks of gestation. Fetal Medicine Foundation First Trimester Screening Group. LANCET. 1998;352(9125):343-6.

13. Chen $Y$, Wang $X$, Lu S, Huang J, Zhang L, Hu W. The diagnostic accuracy of maternal serum alphafetoprotein variants (AFP-L2 and AFP-L3) in predicting fetal open neural tube defects and abdominal wall defects. CLIN CHIM ACTA. 2020;507:125-31.

14. Basbug D, Basbug A, Gulerman C, et al. Is unexplained elevated maternal serum alpha-fetoprotein still important predictor for adverse pregnancy outcome? Ginekol Pol. 2017;88(6):325-30.

15. Endres LK, Krotz S, Grobman WA. Isolated low second-trimester maternal serum beta-human chorionic gonadotropin is not associated with adverse pregnancy outcome. Am J Obstet Gynecol. 2003;189(3):755-7.

16. Onderoglu LS, Kabukcu A. Elevated second trimester human chorionic gonadotropin level associated with adverse pregnancy outcome. Int J Gynaecol Obstet. 1997;56(3):245-9.

17. She BQ, Chen SC, Lee FK, et al. Low maternal serum levels of pregnancy- associated plasma proteinA during the first trimester are associated with subsequent preterm delivery with preterm premature rupture of membranes. Taiwan J Obstet Gynecol. 2007;46(3):242-7. 
18. Faucett AM, Metz TD, DeWitt PE. etal. Effect of obesity on neonatal outcomes in pregnancies with preterm premature rupture of membranes. Am J Obstet Gynecol. 2016;214(2):281-7.

19. Catov JM, Scifres CM, Caritis SN. etal. Neonatal outcomes following preterm birth classified according to placental features. Am J Obstet Gynecol. 2017;216(4):411.

20. Hu J, Zhang J, Chan Y, et al. A rat model of placental inflammation explains the unexplained elevated maternal serum alpha-fetoprotein associated with adverse pregnancy outcomes. J Obstet Gynaecol Res. 2019;45(10):1980-8.

21. Lynch AM, Hart JE, Agwu OC, et al. Association of extremes of prepregnancy BMI with the clinical presentations of preterm birth. Am J Obstet Gynecol. 2014;210(5):421-8.

22. Lim J, Han K, Kim SY, et al. Effects of central obesity on maternal complications in Korean women of reproductive age. Obes Res Clin Pract. 2019;13(2):156-63.

\section{Figures}

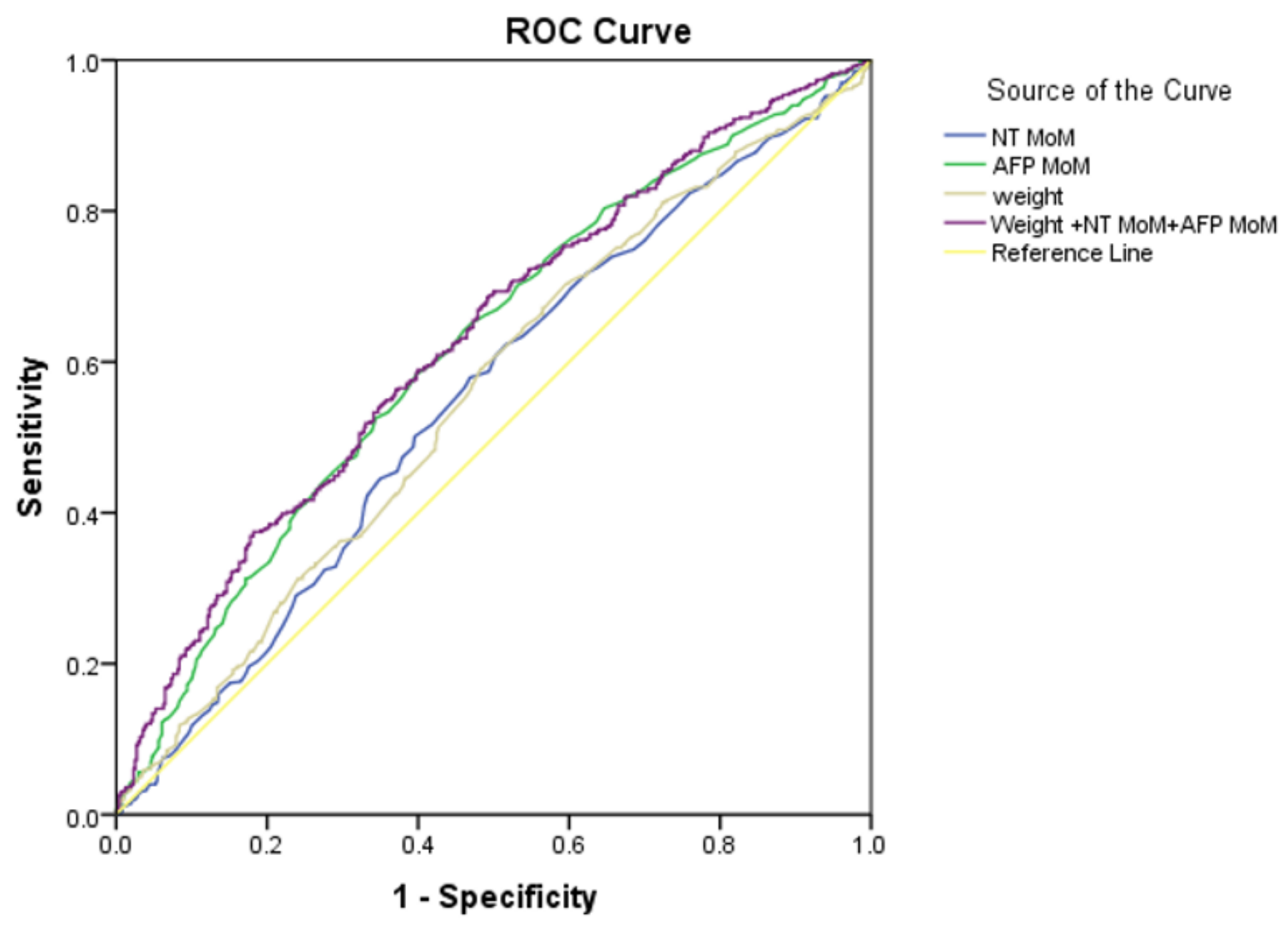

\section{Figure 1}

ROC of AFP, NT and maternal age correspond to MoM in screening PPROM AFP, a-fetoprotein; NT, nuchal translucency; Weight, Maternal weight in the first trimester. 